\title{
Anaemia and organomegaly associated with parasitic infections among schoolchildren in Sengerema District, north-western Tanzania
}

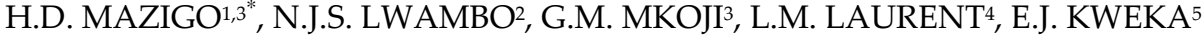 \\ and R. WAIHENYA ${ }^{3}$ \\ ${ }^{1}$ Department of Medical Parasitology E Entomology, Weill-Bugando University College of Health Sciences, \\ P.O. Box 1464, Mwanza, Tanzania \\ ${ }^{2}$ National Institute for Medical Research, P.O. Box 1462, Mwanza, Tanzania \\ 3 Jomo Kenyatta University of Agriculture and Technology, Nairobi, Kenya \\ ${ }^{4}$ Sokoine University of Agriculture, Morogoro, Tanzania \\ 5 Tropical Pesticides Research Institute, Arusha, Tanzania
}

\begin{abstract}
Anaemia and organomegaly are among the health problems affecting schoolchildren in Tanzania and their causes are multifactorial. The objective of this study was to determine the prevalence of anaemia and organomegaly and their relationship with single and multiple parasitic infections among schoolchildren in Sengerema District in north-west Tanzania. This cross sectional study involved 400 schoolchildren. Anaemia and organomegaly were determined using HemoCue photometer and clinical palpation, respectively. A Kato-Katz technique was employed to screen faecal samples for Schistosoma mansoni and other intestinal helminths. Giemsa stained thick and thin blood smears were examined for malaria parasites. The prevalence of anaemia was $19.5 \%(<11 \mathrm{~g} / \mathrm{dl})$ and majority of the children had mild $(22.8 \%)$ to moderate $(36.6 \%)$ anaemia. Organomegaly (palpable spleen and liver) was detected in $41 \%$ of the children and hepatomegaly was the most common (53.7\%). The prevalence of S. mansoni, hookworm and P. falciparum were $64.3 \%$, 38\% and $13.5 \%$ respectively. No significant relationship was observed between single and multiple parasitic infections with anaemia and organomegaly. Logistic regression analysis revealed that increased infections intensity of $S$. mansoni was significantly associated with an increased likelihood of hookworm concomitant infections $(P<0.002)$. In conclusion, the data confirm that malaria, intestinal schistosomiasis and hookworm are common among school children but are not associated with anaemia and organomegaly. Further longitudinal studies are recommended to establish any such association. The prevalence of parasitic co-infections among schoolchildren calls for an integrated control approach to reduce the burden of these infections.
\end{abstract}

Keywords: Anaemia, organomegaly, parasitic infections, schoolchildren, Tanzania

\section{Introduction}

Anaemia is one of the most widespread and common health conditions worldwide. It is estimated to affects $20-50 \%$ of the world's population (Saloojee \& Pettifor, 2001) and is common among children because of nutrition deficiencies, parasitic infections and haemoglobinopathies (Koukounari et al., 2008). Anaemia is estimated to contribute about $23 \%$ of nutrition-related disability-adjusted life years (WHO, 2002). In school age children, anaemia is associated with impairments in physical growth, cognition and school performance (Grantham-McGregor \& Ani, 2001; Koukounari et al., 2008). In the tropics, parasitic infections such as hookworm, schistosomiasis and malaria are predictors for anaemia (Adam et al., 2005; Koukounari et al., 2008; Mahgoub et al., 2009; Bouyou-Akotet et al., 2009). The contribution of single parasitic infections on the risk of anaemia in

\footnotetext{
* Correspondence: Dr. Humphrey D. Mazigo; E-mail: humphreymazigo@gmail.com
} 
schoolchildren is well known, with risk correlated with infection intensity (Stephenson et al., 1985; Friedman et al, 2005). However, little is known about the impact of multiple parasitic infections on anaemia in schoolchildren. The available evidence suggests that co-infections of $P$. falciparum and hookworm are associated with lower haemoglobin than single infections in pre-schoolchildren (Brooker et al., 1999).

Organomegaly is also a common morbidity observed in African schoolchildren and their causes are multifactorial. The contributions of parasitic infections on organ pathology among schoolchildren and adult individuals from various epidemiological settings are well documented (Greenwood, 1987; Fulford et al., 1991; Booth et al., 2004; Malenganisho et al., 2008). Among the manifestations of the high infection intensities of S. mansoni in schoolchildren and adult individuals are hepatomegaly and splenomegaly (Vennervald \& Dunne, 2004; Booth et al., 2004; Malenganisho et al., 2008). Chronic exposure to malaria also causes overlapping physical signs of hepatomegaly or splenomegaly in school-aged children (Greenwood, 1987; Booth et al., 2004). Studies have reported the synergistic effects of plasmodia and schistosomes co-infection on the organ pathology but most of the results are not conclusive (Fulford et al., 1991; Mwatha et al., 2003; Booth et al., 2004; Vennervald et al., 2005).

In Tanzania, anaemia, organomegaly and parasitic infections have been reported to be common among schoolchildren (Lwambo et al., 1999, 2000; Mboera et al., 2007), but studies on the contributions of single or multiple parasitic infections on these morbidities are limited. Understanding the contributions of single or multiple parasitic infections on anaemia and organomegaly in schoolchildren, will serve in designing better integrated approaches for disease management. The present study was therefore, conducted to determine the prevalence of anaemia, organomegaly and to relate these morbidities with single and multiple parasitic infections among schoolchildren (aged 8-16 years) in Sengerema District in northwest Tanzania. In addition, the associations between different infection intensities of hookworm and S. mansoni were also determined.

\section{Material and methods}

\section{Study area and population}

The cross sectional study was carried out in Nyamatongo Ward, Sengerema district, northwest Tanzania from March to May 2009. The Ward is located on the eastern part of the district and it borders Lake Victoria to the north and east. The area experiences a long rainy season between February-May and short rainy season from September-December. The area receives an annual precipitation of $930-1,200 \mathrm{~mm}$ the mean maximum temperature ranges from $25^{\circ} \mathrm{C}-28^{\circ} \mathrm{C}$ (September-December) and the mean low temperatures ranges from $11^{\circ} \mathrm{C}$ $20^{\circ} \mathrm{C}$ (June-August). The inland area contains a number of small seasonal rivers. The main occupations of the residents of the Ward include crop production (rice and vegetables), fishing and livestock keeping.

The study involved schoolchildren attending four primary schools namely, Nyalwambu, Karumo, Kamanga and Irunda. All the schools were at a distance of $05-1.5 \mathrm{~km}$ from the Lake Victoria shore. The inclusion criteria for the study subject were as follows: children age ranged between 8-16 years who provided written informed assent from their parents or guardians, and who were permanently living in the study area. On the other hand, children were excluded if they didn't meet the stated inclusion criteria. A Simple Random Sampling method was used to select children to participate in the study. Using the 
class attendance register, each child was given a number and selection of the children to participate in the study was achieved by using tables of random numbers.

\section{Parasitological and morbidity survey}

A single stool sample was collected from each child in labelled containers and processed using duplicate Kato-Katz thick smears (Katz et al., 1972). The intensity of infection was estimated indirectly by counting the number of eggs per gram of faeces (epg). The smears were examined within 30-60 minutes for hookworm characteristics eggs and examined the following day for Schistosoma mansoni, Ascaris lumbricoides and Trichuris trichiura.

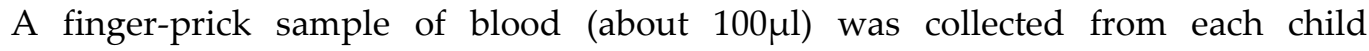
submitted faecal sample and used to measures venous haemoglobin $(\mathrm{Hb})$, in a HemoCue photometer (HemoCue, Angelholm, Sweden) (von Schenck et al. 1986) and both thick and thin Giemsa stained blood smears were prepared for malaria parasites identification and quantification, respectively (Cheesbrough, 1998). All children who provided blood and faecal samples were also examined clinically by palpation by two qualified physicians for enlarged livers and spleens in supine position (Vennervald et al., 2004). Liver and spleen were considered enlarged, if they were palpable $>2 \mathrm{~cm}$ below the costal line and the clinical findings were classified into (i) No organomegaly; (ii) soft organomegaly (liver/spleen); (iii) firm/hard splenomegaly; (iv) firm/hard hepatomegaly; and (v) firm/hard hepatosplenomegaly.

\section{Ethical consideration}

The study was conduct using protocol approved by the Publications and Research Committee (certificate no. BREC/001/03/2009) of the Weill-Bugando University College of Health Sciences and Bugando Medical Centre, Mwanza, Tanzania. All children found positive for hookworm and S. mansoni infections were treated with mebendazole $500 \mathrm{mg}$ and praziquantel $40 \mathrm{mg} / \mathrm{kg}$ (WHO, 2005). Individuals found positive for malaria were referred to the nearby health centres, where they were given artemisin-based combination therapy (ACT) on an outpatient basis. Anaemic children received ferrous sulphate tablets (hematinics, Shelys Pharmaceuticals Ltd, Tanzania).

\section{Data analysis}

Data from each school child were recorded into the questionnaire and thereafter entered twice in database created in MS Access for validation and then analyzed using SPSS for window version 11.5 (SPSS Inc, Chicago, IL, USA). Arithmetic mean egg counts from two Kato-Katz thick smears were calculated for S. mansoni and hookworm. Infections intensity of S. mansoni and hookworm were classified according to WHO criteria (WHO, 1991; WHO, 2005). Plasmodium falciparum infection intensities were stratified into four categories: 1-50, 51$500,501-5000$ and $>5000$ parasites/ $\mu \mathrm{L}$ of blood.

The prevalence of anaemia and organomegaly were stratified by age groups $(\leq 10,11$ 13 and 14-16 years), gender and compared using Pearson $\chi^{2}$ analysis. Children with anaemia and organomegaly were also grouped according to infections intensities of S. mansoni, hookworm, P. falciparum and compared between sex and age. The associations between anaemia and organomegaly with parasitic infections were analyzed using Pearson $\chi^{2}$. Children found to have $<11 \mathrm{~g} / \mathrm{dL}$ were considered anaemic, with $\mathrm{Hb}$ concentrations of $<7 \mathrm{~g} / \mathrm{dL}, 7.0-9.9 \mathrm{~g} / \mathrm{dL}, 10.0-10.9 \mathrm{~g} / \mathrm{dL}$ and $\geq 11 \mathrm{~g} / \mathrm{dL}$ indicating severe anaemia, mild, moderate 
and normal (WHO, 2001). Logistic regression and $\chi^{2}$-test for trend were done to investigate whether there was any association between hookworm and S. mansoni infection intensity.

\section{Results}

\section{Prevalence of parasitic infections}

A total of 400 schoolchildren were involved in the study. There were more girls $(n=204)$ than boys ( $\mathrm{n}=196)$. The mean age of the children was 12.2 years, with a range between 8-16 years and $52.5 \%(n=210)$ of them were aged between $11-13$ years. Of 400 children, 218/400 was infected with at least one parasite species. S. mansoni was more prevalent $(64.3 \%)$ than $P$. falciparum (13.5\%) and hookworm (38\%). T. trichiura and A. lumbricoides were absent. The overall prevalence of double and triple parasitic infection was $26.5 \%$ and $2.8 \%$, respectively.

\section{Prevalence of anaemia}

The mean haemoglobin $(\mathrm{Hb})$ of the schoolchildren was $12.1 \mathrm{~g} / \mathrm{dL}$ (range 7.1-16.7/dL). The mean $\mathrm{Hb}$ in males and females was $12.1 \pm 0.543 \mathrm{~g} / \mathrm{dL}$ and $12.9 \pm 0.624 \mathrm{~g} / \mathrm{dL}$, respectively and the difference was significant $\left(\chi^{2}=14.485, P<0.006\right)$. About $20 \% \quad(n=79 / 400)$ of the schoolchildren were anaemic $(<11 \mathrm{~g} / \mathrm{dL})$ and $50.6 \%(40 / 79)$ of the anaemic children were in the age group $11-13$ years (Table 1 ). Importantly, $22.8 \%$ and $38.6 \%$ of the children had mild and moderate anaemia, respectively (Table 1 ).

Table 1: Prevalence of anaemia (\%) among schoolchildren by age and sex

\begin{tabular}{|c|c|c|c|c|c|}
\hline Variable & $\begin{array}{c}\text { Mild } \\
7.0-9.9 \mathrm{~g} / \mathrm{dL}\end{array}$ & $\begin{array}{l}\text { Moderate } \\
10.0-10.9 \mathrm{~g} / \mathrm{dL}\end{array}$ & $\begin{array}{l}\text { Normal } \\
\geq 11 \mathrm{~g} / \mathrm{dL}\end{array}$ & $\chi^{2}$ & $P$-value \\
\hline \multicolumn{6}{|l|}{ Sex } \\
\hline Female & $13(6.4 \%)$ & $22(10.8 \%)$ & $169(82.8 \%)$ & 14.49 & 0.006 \\
\hline Male & $17(8.7 \%)$ & $27(13.8 \%)$ & $152(77.6 \%)$ & & \\
\hline \multicolumn{6}{|c|}{ Age (years) } \\
\hline$\leq 10$ & $8(9.6 \%)$ & 14 (16.9\%) & $61(73.5 \%)$ & 3.60 & 0.462 \\
\hline $11-13$ & $16(7.6 \%)$ & $24(11.4 \%)$ & $170(80.9 \%)$ & & \\
\hline $14-17$ & $6(5.6 \%)$ & $11(10.3 \%)$ & $90(84.1 \%)$ & & \\
\hline
\end{tabular}

\section{Anaemia in relation to parasitic infections}

Of all 54 children diagnosed with $P$. falciparum, only $3.7 \%(\mathrm{n}=2 / 54)$, were anaemic $(<11 \mathrm{~g} / \mathrm{dl})$ with only 1 child having a parasitaemia of $<5,000$ parasites/ $\mu \mathrm{L}$. Two children had parasitaemia of $<5,000$ parasites $/ \mu \mathrm{L}$ and were not anaemic. However, no relationship was observed between intensity of $P$. falciparum infection and mean haemoglobin levels of the infected children $\left(\chi^{2}=5.834, P<0.67\right)$. On the other hand, of the 257 children infected with $S$. mansoni, $12.5 \%(\mathrm{n}=32 / 257)$ were anaemic $(<11 \mathrm{~g} / \mathrm{dl})$ and majority of the anaemic individuals had light to moderate egg intensity. About $87.5 \%(n=203)$ of the children with $S$. mansoni infection were not anaemic. There was no relationship between the mean haemoglobin level and intensity of $S$. mansoni infection.

Of 152 children infected with hookworms, 9.2\% ( $n=14 / 152)$ were anaemic. Two individuals had heavy infection intensity ( $\geq 4,000 \mathrm{eggs} / \mathrm{g}$ faeces) and were anaemic. The mean haemoglobin concentration of the children with hookworm infection was observed to decrease with increase in egg intensity, children with $\geq 4,000 \mathrm{epg}$ had, on average mean $\mathrm{Hb}$ 
$<11 \mathrm{~g} / \mathrm{dl}$. There was no associations between hookworm infection intensity and anaemia or haemoglobin concentration $\left(\chi^{2}=1.405, P<0.704\right)$.

A total of $106(26.5 \%)$ of the children had parasitic co-infections and 20\% ( $n=21 / 106)$ of them were anaemic. About $14.2 \%$ were co-infected with S. mansoni + hookworm, $4.7 \%$ with S. mansoni + P. falciparum and $0.9 \%$ with hookworm + P. falciparum. About $77 \%$ $(n=82 / 106)$ of the children had dual parasitic infection and were not anaemic. Only 2 out of 11 children with triple infections (S. mansoni + hookworm $+P$. falciparum) were anaemic $(<11 \mathrm{~g} / \mathrm{dl})$. In either case, no relationship was observed between double or triple parasitic infections with anaemia or mean haemoglobin.

\section{Prevalence of organomegaly}

In general, $41 \%(164 / 400)$ of the children had organomegaly. The prevalence of hepatomegaly, splenomegaly and hepatosplenomegaly was 53.7\% (88/164), 28\% (46/164) and $18.3 \%(30 / 154)$ respectively (Table 2$)$. The prevalence of hepatomegaly and splenomegaly was higher in male than in females and the sex-related difference was significant $(P<0.008)$. Significantly more males $(11.2 \%)$ than females $(3.9 \%)$ had hepatosplenomegaly $\left(\chi^{2}=7.685, P<\right.$ 0.006) (Table 2). Overall, children 11-13 years old had a higher prevalence of splenomegaly, hepatomegaly and hepatosplenomegaly (Table 3$)$. When compared with other age groups, no significance difference was observed $\left(\chi^{2}=1.637, P<0.373\right)$.

Table 2: Frequency of organomegaly among schoolchildren in Nyamatongo ward by sex

\begin{tabular}{lclll}
\hline Variable & Female $(\mathbf{N}=\mathbf{2 0 4})$ & Male $\mathbf{( N = 1 9 6 )}$ & $\boldsymbol{\chi}^{\mathbf{2}}$ & P-value \\
\hline Hepatomegaly & $36(17.7 \%)$ & $52(26.5 \%)$ & 4.597 & $<0.032$ \\
Splenomegaly & $15(7.4 \%)$ & $31(15.8 \%)$ & 7.035 & $<0.008$ \\
Hepatosplenomegaly & $8(3.9 \%)$ & $22(11.2 \%)$ & 7.685 & $<0.006$ \\
Total organomegaly & $59(29 \%)$ & $105(53.5 \%)$ & 19.317 & $<0.046$ \\
\hline
\end{tabular}

\section{Organomegaly in relation to parasitic infections}

Of the 54 children infected with $P$. falciparum, 16.6\% (9/54), 7.4\% (4/54) and 5.6\% (3/54) had hepatomegaly, splenomegaly and hepatosplenomegaly, respectively. No significant relationship was observed between intensity of $P$. falciparum infections and either of the organomegaly $\left(\chi^{2}=4.85, P<0.73\right)$. Of 257 children with $S$. mansoni eggs in their stool samples, $23 \%(59 / 257), 9.3 \%(25 / 257)$ and $6.2 \%(16 / 257)$ had hepatomegaly, splenomegaly and hepatosplenomegaly, respectively. However, no significant association was observed between $S$. mansoni infections intensity and organomegaly $\left(\chi^{2}=4.05, P<0.39\right)$. Children having single infections of hookworm were also having organomegaly. About, 23.7\% (36/152), $13.8 \%(21 / 152)$ and $11 \%(11 / 152)$ of the children with hookworm infections had hepatomegaly, splenomegaly, and hepatosplenomegaly.

Table 3: Prevalence of hepatomegaly, splenomegaly and hepatosplenomegaly among schoolchildren

\begin{tabular}{lcccccc}
\hline Variable & $\begin{array}{l}\text { Age group } \\
\text { Overall }(95 \% \mathrm{CI})\end{array}$ & $\leq 10$ & $11-13$ & $14-16$ & $\chi^{2}$ & P-value \\
\hline Hepatomegaly & $22(17.4-26.1)$ & 9 & 21 & 16 & 1.753 & 0.416 \\
Splenomegaly & $11.5(8.4-14.6)$ & 14 & 52 & 22 & 2.337 & 0.311 \\
Hepatosplenomegaly & $7.5(4.92-10.1)$ & 5 & 16 & 9 & 0.822 & 0.393 \\
Total organomegaly & 40.5 & 28 & 89 & 47 & 4.912 & 1.12 \\
\hline
\end{tabular}


Children co-infected with $S$. mansoni and hookworm had higher prevalence of hepatomegaly $(16 \%)$ as compared to children co-infected with S. mansoni and P. falciparum (3.8\%) $(P<0.003)$. Only 2 children were co-infected with 3 parasites (S. mansoni + P. falciparum + Hookworm) had hepatomegaly and the rest had hepatosplenomegaly or splenomegaly (Table 4). No significant associations were observed between parasites co-infections and organomegaly $\left(\chi^{2}=0.07, P<0.8\right)$.

\section{Anaemia and organomegaly in relation to parasitic infections}

About $19 \%(6 / 32), 3.1 \%(1 / 32)$ and $13 \%(4 / 32)$ of the anaemic children infected with $S$. mansoni had hepatomegaly, splenomegaly, and hepatosplenomegaly. Of the anaemic children infected with hookworm, 21\% (3/14), 7.1\% (1/14) and 7.1\% (1/14) had hepatomegaly, splenomegaly and hepatosplenomegaly, respectively. None of the anaemic children infected with P. falciparum had any type of organomegaly. Only 1 child among those co-infected with S. mansoni and P. falciparum (1/4) had hepatomegaly. Only one among the anaemic children was found with an enlarged spleen without any of the three parasitic infections. Organomegaly was not observed in $6(40 \%)$ of 15 anaemic children infected with both $S$. mansoni + hookworm. Similarly, organomegaly was not observed among 3 of the 4 children co-infected with $S$. mansoni $+P$. falciparum. All children co-infected with hookworm + P. falciparum had no organomegaly.

Table 4: Number (\%) of children with organomegaly co-infected with two or three parasites

\begin{tabular}{lcccccccc}
\hline Variable & Sm+Hk & \multicolumn{3}{c}{ Sm+P.f } & \multicolumn{3}{c}{ Pf+Hk } & \multicolumn{2}{c}{ Sm+Pf+Hk } \\
\hline Splenomegaly & 10 & $(9.4)$ & 1 & $(0.9)$ & 1 & $(0.9)$ & 1 & $(9.1)$ \\
Hepatomegaly & 17 & $(16)$ & 4 & $(3.8)$ & 1 & $(0.9)$ & 2 & $(18)$ \\
Hepatosplenomegaly & 4 & $(3.8)$ & 1 & $(0.9)$ & 0 & $(0)$ & 1 & $(9.1)$ \\
Total & 31 & $(29.2)$ & 6 & $(5.6)$ & 2 & $(1.8)$ & 4 & $(36.4)$ \\
\hline
\end{tabular}

Key: $\mathrm{Sm}=$ S. mansoni, Hk= Hookworm, Pf= P. falciparum)

\section{Relationship between hookworm infections and infection intensities of S. mansoni}

Increase in $S$. mansoni infection intensity was strongly associated with the likelihood of concomitant infections with hookworm $(P<0.002)$. Thus, children with moderate (101400epg) and heavy (>400epg) S. mansoni infection intensities were likely to be infected with hookworm. High odd ratios (OR) were observed when the number of hookworm infections was compared between $S$. mansoni-negative individuals and those with either a moderate $(\mathrm{OR}=2.12,(95 \% \mathrm{CI}, 1.13-3.99), P<0.002)$ or a heavy $S$. mansoni infection intensities $(\mathrm{OR}=2.18$, (95\%CI, 1.22-3.90), $P<0.01)$.

Table 5: Number of schoolchildren with or without hookworm infections in relation to different infection intensities of $S$. mansoni

\begin{tabular}{llllll}
\hline & Hookworm & & & & \\
S. mansoni infections status & Positive & Negative & Odd ratio & $\mathbf{9 5 \%}$ CI & $\boldsymbol{P}$-value \\
\hline Negative (0 epg) & 64 & 79 & 1 & & \\
Light infection (1-100epg) & 41 & 52 & 1.17 & $0.60-2.32$ & 0.639 \\
Moderate infection (101-400epg) & 24 & 55 & 2.12 & $1.13-3.99$ & 0.02 \\
Heavy infection > 400 epg & 23 & 62 & 2.18 & $1.22-3.90$ & 0.01 \\
\hline
\end{tabular}




\section{Discussion}

The present study demonstrated that anaemia is still a problem among schoolchildren in Tanzania (Partnership for Child Development, 1998; Lwambo et al., 2000; Mboera et al., 2007). The prevalence of anaemia observed in our study was lower that in a neighbouring district of Magu district (62.6\%) (Lwambo et al., 2000) and other places in Tanzania (Tatala et al., 2008). One explanation for this difference could be variations in nutrition status or risks factors for anaemia.

Hookworm, schistosomiasis and malaria are major causes of anaemia (Sturrock et al., 1966; Olsen et al., 1998; Tatala et al., 2008; Koukounari et al., 2008) with hookworm accounting for up to $35 \%-73 \%$ of the iron-deficiency anaemia in Africa (Stoltzfus et al., 1997). In this study, the mean $\mathrm{Hb}$ levels were observed to decrease with increase in hookworm egg intensity. A similar observation was reported by Olsen et al., (1998) in Western Kenya. However, no association was observed between hookworm infection and anaemia in this study. The lack of association is probably due to the relatively low intensity of hookworm infection in the study population. In the present study, anaemic children were infected with S. mansoni. Unlike in our study where no relationship was observed between mean $\mathrm{Hb}$ and intensity of $S$. mansoni infection, in Uganda children heavily infected with $S$. mansoni were found to have significantly lower $\mathrm{Hb}$ counts compared with those not infected (Koukounari et al., 2006). The infection intensities in the present study area were probably too low to have a significant effect on haemoglobin and hence anaemia.

In this study, anaemic children were infected with $P$. falciparum. Malaria infection contributes to reduced haemoglobin concentrations through a number of mechanisms (Philips \& Pasvol, 1992). In the present study, no correlations were observed between mean haemoglobin and $P$. falciparum parasitaemia. A similar observation has been reported among schoolchildren in Zanzibar (Stoltzfus et al., 1997) where it was suggested that asymptomatic malarial infection did not influence $\mathrm{Hb}$. Alternative explanation for this could be that, in endemic areas, children suffer from several malaria attacks which may decrease their $\mathrm{Hb}$ to low levels, but the time interval between one malaria episode and another is sufficient to restore $\mathrm{Hb}$ values, resulting in a lack of fluctuation in response to parasitaemia.

Although co-infections of $P$. falciparum, S. mansoni and hookworms have been described to enhance the severity of anaemia because of the distinct mechanisms with which each parasite causes anaemia (Mwangi et al., 2006), in our study multiple parasitic infections were not associated with anaemia. In the present study, only few children had triple infection and in any case anaemia levels were mild to moderate. In contrary, Sturrock (1996) reported associations of hookworm and malaria with hypochromic anaemia from a number of studies in East Africa.

Our findings on organomegaly indicate that, male individuals had higher prevalence of hepatomegaly and splenomegaly than females and the age group 11-13 years having higher prevalence of hepatomegaly. A similar observation has been reported from Makueni district, Kenya (Vennervald \& Dunne, 2004). Schistosoma mansoni and malaria are major cause of organomegaly in African children (Vennervald et al., 2004; Grobusch \& Kremsner, 2005). Thus, it is possible therefore that co-infection with Schistosoma and Plasmodium species may have synergistic effects on organ pathology. However, in the present study, no direct correlation was observed between hepatomegaly, splenomegaly or hepatosplenomegaly with infection intensities in the case of S. mansoni, P. falciparum or co-infections. Similar findings have been reported in Kenya (Wilson et al., 2007). 
In our study, anaemic children infected with S. mansoni, hookworm and P. falciparum were also observed to have hepatomegaly, splenomegaly and hepatosplenomegaly. Splenomegaly is known to be associated with anaemia, possibly because of sequestration of red blood cells in the spleen which reduces the effective circulating mass of red blood cells (Kloos et al., 1997). However, no correlation was observed between splenomegaly and anaemia in the present study.

Organomegaly was also detected in children without S. mansoni or P. falciparum infections. Likewise, anaemia was observed in children with no any of the two parasitic infections, suggesting that, there may be other causes of anaemia in the study area like elsewhere in Sub-Saharan Africa (Sturrock et al., 1996; Olsen et al., 1998; Lwambo et al., 2000).

In this study, we found association between S. mansoni and hookworm infections intensities. Children with an increasing infection intensity of S. mansoni were more likely to be concurrently infected with hookworms. This observation was not unusual, similar findings have been reported in Cote d'Ivoire (Keiser et al., 2002), but in contrast to studies from Mali and Tanzania (De Clearq et al., 1995; Booth et al., 1998). Variations in suitable conditions for transmissions of schistosomes and hookworm could partly explain the differences observed.

There are a number of limitations in this study. The study did not investigate other factors associated with anaemia such as other parasitic infections, iron deficiency, micronutrient deficiencies and haemoglobinopathy. Furthermore, the study used only clinical palpation method to diagnose organomegaly which was obvious only in children with obvious signs and missed those with mild signs. Thus, the use of ultrasonography is important. Further investigation within risk groups and longitudinal studies are needed to determine the role of multiple parasitic infections and other pre-disposing factors in the development and outcomes of anaemia and organomegaly.

In conclusion, the results of significant associations of S. mansoni and hookworm infection intensities stress the importance of considering the joint impact of multiple-species when addressing the health of schoolchildren. This may aid in designing appropriate managements strategies for the control of these significant public health problems.

Received 30 December 2009

Revised 24 March 2010

Accepted 25 March 2010

\section{References}

Adam, I., Khamis, A.H. \& Elbashir, M.I. (2005) Prevalence and risk factors for anaemia in pregnant women of eastern Sudan. Transactions of the Royal Society of Tropical Medicine and Hygiene 99, 739-743

Booth, M., Mayombana, C., Machibya, H., Masanja, H., Odermatt, P., Utzinger, J. \& Kilima, P. (1998) The use of morbidity questionnaires to identify communities with high prevalences of schistosome or geohelminth infections in Tanzania. Transactions of the Royal Society of Tropical Medicine and Hygiene 92, 484-490.

Booth, M., Vennervald, B.J. \& Kent, L. (2004) Micro-geographical variation in exposure to Schistosoma mansoni and malaria, exacerbation of splenomegaly in Kenyan schoolchildren. BMC Infectious Disease 4, 13. 
Bouyou-Akotet, M.K, Dzeing-Ella, A., Kendjo, E., Etoughe, D., Ngoungou, E.B., Planche, T., Koko, J. \& Kombila, M. (2009).Impact of Plasmodium falciparum infection on the frequency of moderate to severe anaemia in children below 10 years of age in Gabon. Malaria Journal 2009, 8:166 doi:10.1186/1475-2875-8-166

Brooker, S., Peshu, N., Warn, P., Mosobo, M., Guyatt, H., Marsh, K. \& Snow, R. (1999) Epidemiology of hookworm infection and its contribution to anaemia among preschoolchildren on the Kenyan coast. Transactions of the Royal Society of Tropical Medicine and Hygiene 93, 240-246.

Cheesbrough, M. (1998) Medical Laboratory Manual for Tropical Countries. $5^{\text {thed. Cambridge: }}$ Heinemann Ltd.

De Clearq, D., Sacko, M., Behnke, J.M., Traore, M. \& Vercruysse, J. (1995) Schistosoma and geohelminth infections in Mali, West Africa. Annals of the Belgian Society of Tropical Medicine 75, 191-199.

Friedman, J.F., Kanzaria, H.K. \& McGarvey, S.T. (2005) Human schistosomiasis and anaemia: the relationship and potential mechanism. Trends in Parasitology 21, 386-392.

Fulford, A.J., Mbugua, G.G., Ouma, J.H., Kariuki, H.C., Sturrock, R.F. \& Butterworth, A.E. (1991). Differences in the rate of hepatosplenomegaly due to Schistosoma mansoni infection between two areas in Machakos District, Kenya. Transactions of the Royal Society of Tropical Medicine and Hygiene 85, 481-488.

Grantham-McGregor, S. \& Ani, C. (2001) A review of studies on the effect of iron deficiency on cognitive development in children. Journal of Nutrition 131:649S-666S.

Greenwood, B.M. (1987). Asymptomatic malaria infections do they matter? Parasitology Today, 3, 206-214.

Grobusch, M.P. \& Kremsner, P.G. (2005) Uncomplicated malaria. Current Topics in Microbiology and Immunology, 295, 83-104.

Katz, N., Chaves, A. \& Pellegrino,J. (1972). A simple device for quantitative stool thicksmear technique in schistosomiasis mansoni. Revista do Instituto Medicina Tropical de Sao Paulo 14, 397-400.

Keiser, J., N'Goran, E.K., Singer, B.H., Lengeler, C., Tanner, M. \& Utzinger, J. (2002) Association between Schistosoma mansoni and hookworm infections among schoolchildren in Cote d'Ivoire. Acta Tropica 84, 31-41.

Kloos, H., Fulford, A.J., Butterworth, A.E., Sturrock, R.F., Ouma, J.H., Kariuki, H.C., Thiongo, F.W., Dalton, P.R. \& Klumpp, R.K. (1997) Spatial patterns of human water contact and Schistosoma mansoni transmission and infection in four rural areas in Machakos District, Kenya. Social Science and Medicine 44, 949- 968.

Koukounari, A., Estambale, B.B., Njagi, J.K., Cundill, B., Ajanga, A., Crudder, C., Otido, J., Jukes, M.C., Clarke, S.E. \& Brooker, S. (2008). Relationships between anaemia and parasitic infections in Kenyan schoolchildren: a Bayesian hierarchical modelling approach. International Journal of Parasitology 38, 1663-1671

Koukounari, A., Fenwick, A., Whawell, S., Kabatereine, N.B., Kazibwe, F., Tukahebwa, E.M., Stothard, J.R., Donnelly, C.A. \& Webster, J.P. (2006) Morbidity indicators of Schistosoma mansoni: Relationship between infection and anemia in Ugandan schoolchildren before and after praziquantel and albendazole chemotherapy. American Journal of Tropical Medicine and Hygiene 75, 278-286

Lwambo, N.J.S., Brooker, S., Siza, J.E., Bundy, D.A.P. \& Guyatt, H. (2000) Age patterns in stunting and anaemia in African schoolchildren: a cross-sectional study in Tanzania. European Journal of Clinical Nutrition 54, 36-40. 
Lwambo, N.J.S., Siza, J.E., Brooker, S., Bundy, D.A.P. \& Guyatt, H. (1999) Patterns of concurrent hookworm infection and schistosomiasis in schoolchildren in Tanzania. Transactions of the Royal Society of Tropical Medicine and Hygiene 93, 497-502.

Mahgoub, H.M., Mohamed, A.A., Magzoub, M., Gasim, G.I., Eldein, W.N., Ahmed, A.A. \& Adam, I. (2009). Schistosoma mansoni infection as a predictor of severe anaemia in schoolchildren in eastern Sudan. Journal of Helminthology 28, 1-4.

Malenganisho, W.L.M., Magnussen, P., Friis, H., Siza, J., Kaatano, G., Temu, M., Vennervald, B.J. (2008). Schistosoma mansoni morbidity among adults in two villages along Lake Victoria shores in Mwanza District, Tanzania. Transactions of the Royal Society of Tropical Medicine and Hygiene, 102:532-541

Mboera, L.E.G., Mlozi, M.R.S., Senkoro, K.P., Rwegoshora, R.T., Rumisha, S.F., Mayala, B.K., Shayo, E.H., Senkondo, E., Mutayoba, B., Mwingira, V. \& Maerere, A. (2007) Malaria and Agriculture in Tanzania: impact of land use and Agricultural Practices on Malaria Burden in Mvomero District. National Institute for Medical Research, Dar es Salaam, Tanzania.

Mwangi, T., Bethony, J. \& Brooker, S. (2006) Malaria and helminths interactions in humans: an epidemiological viewpoint. Annals of Tropical Medicine and Parasitology, 100:7:551570 .

Mwatha, J.K., Jones, F.M. \& Mohamed, G. (2003) Associations between anti-Schistosoma mansoni and anti-Plasmodium falciparum antibody responses and hepatomegaly, in Kenyan schoolchildren. Journal of Infectious Diseases 187, 1337-1341.

Olsen, A., Magnussen, P., Ouma, J.H., Andreassen, J. \& Friis, H. (1998). The contribution of hookworm and other parasitic infections to haemoglobin and iron status among children and adults in Western Kenya. Transactions of the Royal Society of Tropical Medicine and Hygiene, 92:643-649

Partnership for Child Development (1998). The anthropometric status of schoolchildren: in five countries in the Partnership for Child Development. Proceedings of Nutrition Society 57, 149-158.

Saloojee, H. \& Pettifor, J.M. (2001). Iron deficiency and impaired child development. British Medical Journal 323, 1377-1378.

Stephenson, L.S., Latham, M.C., Kurz, K.M., Kinoti, S.N., Oduori, M.L. \& Crompton, D.W. (1985). Relationships of Schistosoma haematobium, hookworm and malaria infections and metrifonate treatment to haemoglobin level in Kenyan schoolchildren. American Journal of Tropical Medicine and Hygiene 34, 519-528.

Sturrock, R.F. (1966) Observation on the associations of hookworm and malaria with hypochromic anaemia in four rural communities in East Africa. East African Medical Journal 43, 603-609.

Sturrock, R.F., Kariuki, H.C., Thiongo, F.W., Gachare, J.W., Omondi, B.G.O., Ouma, J.H., Mbugua, G. \& Butterworth, A.E. (1996) Schistosomiasis mansoni in Kenya: relationship between infection and anaemia in schoolchildren at the community level. Transactions of the Royal Society of Tropical Medicine and Hygiene 90, 48-54.

Tatala, S.R., Kihamia, C.M., Kyungu, L.H. \& Syanberg, H. (2008) Risk factors for schoolchildren in Tanga Region, Tanzania. Tanzania Journal Health Research 10, 189202.

Vennervald, B.J. \& Dunne, D.W. (2004) Morbidity in schistosomiasis: an update. Current Opinion in Infectious Diseases 17, 439-447. 
Vennervald, B.J., Booth, M. \& Butterworth, A.E. (2005) Regression of hepatomegaly in Kenyan school-aged children after praziquantel treatment and three years of greatly reduced exposure to Schistosoma mansoni. Transactions of the Royal Society of Tropical Medicine and Hygiene 99, 150-160.

Vennervald, B.J., Kenty, L. \& Butterworth, A.E. (2004) Detailed clinical and ultrasound examination of children and adolescents in a Schistosoma mansoni endemic area in Kenya: hepatosplenic disease in the absence of portal fibrosis. Tropical Medicine and International Health 9, 461-470.

von Schenck, H., Falkensson, M. \& Lundberg, B. (1986) Evaluation of "HemoCue," a new device for determining hemoglobin. Clinical Chemistry 3, 526-529.

WHO (1991) Hookworm Infection and Anaemia: Approaches to Prevention and Control. World Health Organization, Geneva.

WHO (2001) Iron Deficiency Anaemia. Assessment, Prevention and Control. A guide for Programme Managers. World Health Organization, Geneva.

WHO (2002). Reducing Risks, Promoting Healthy Life. The World Health Report 2002. World Health Organization, Geneva.

WHO (2005) Deworming for health and development. Report of the Third Global Meeting for Partners for Parasite Control. Geneva; World Health Organization

Wilson, S.,Vennervald, B.J.,Hadzo, H., Ireri, E., Amaganga, C., Booth, M., Kariuki, H.C., Mwatha, J.K., Kimani, G., Ouma, J.H., Muchiri, E. \& Dunne, D.W. (2007) Hepatosplenomegaly in Kenyan schoolchildren: exacerbation by concurrent chronic exposure to malaria and Schistosoma mansoni infection. Tropical Medicine and International Health 12, 1442-1449. 